\title{
A study on the semi-active suspension systems used for motor vehicles
}

\author{
Cătălin Alexandru ${ }^{1}$ \\ ${ }^{1}$ Product Design, Mechatronics and Environment Department, Transilvania University of \\ Braşov, Romania \\ E-mail: calex@unitbv.ro
}

\begin{abstract}
This work deals with a study on the semi-active suspension systems used for motor vehicles, with focus on the devices that control the suspension damping. Several representative types of semi-active devices are presented and then analyzed comparatively using a multicriteria method based on the FRISCO formula. Specific control techniques are then discussed, emphasizing the application field and their limitations. A novel control strategy is proposed with the aim to improve the dynamic behaviour of the vehicle. The mechatronic model of the semi-active suspension (which integrates mechanical and control components) was carried out and simulated in a virtual environment by using MBS (Multi-Body Systems) and DFC (Design for Control) software solutions, namely ADAMS and EASY5 of MSC Software.
\end{abstract}

Keywords: semi-active suspension systems, motor vehicles, mechatronic model

\section{Introduction}

Once with increasing the running speeds, equipping cars with increasingly evolved suspension systems, capable of creating a barrier of vibrations and noises between wheels and car body has become a necessity, all the more so since the speed on uneven roads is rather limited by the suspension quality than the performance of the propulsion system. The advances in microelectronics, sensor technology, and actuating systems have enabled the design of adaptive suspension systems (computercontrolled) that provide a better dynamic behaviour by reference to the classical ones $[1,2,4,13,14$, 17, 23, 27]. From this perspective, three types of suspension systems can be systematized (Figure 1):

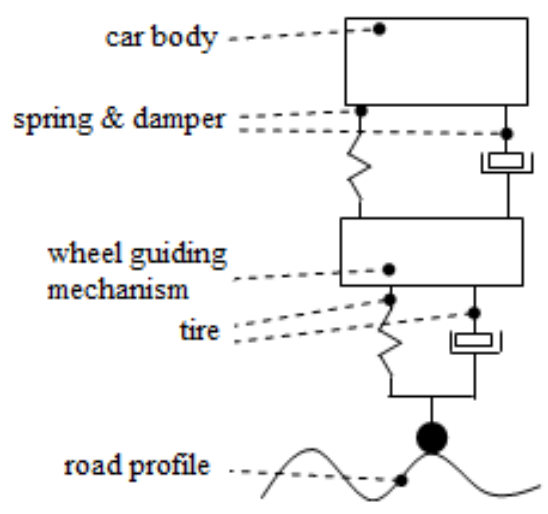

a.

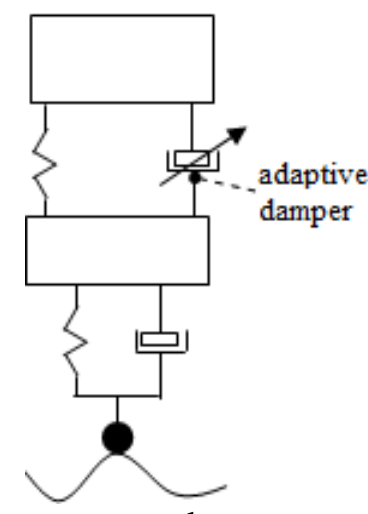

b.

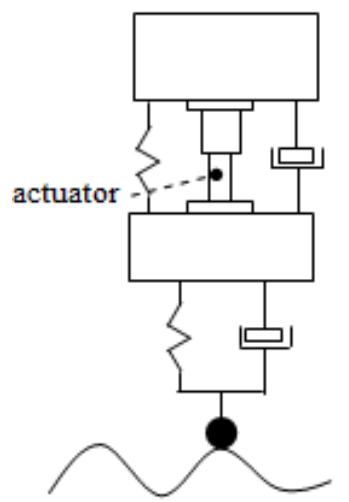

c.

Figure 1. Basic types of suspension systems. 
(a) passive systems - their dynamic behavior is defined by the characteristics of the elastic and damping elements, and it cannot be changed during operation;

(b) semi-active systems - their operation is based on changing the suspension characteristics (e.g. damping, inertia) without introducing external forces in the system;

(c) active systems - their operation is based on changing the system behaviour by applying external forces, which are usually generated by linear actuators.

The semi-active solution can provide dynamic behaviour performances close to that of the active suspension, but with lower energy resources (because it does not inject energy into the system). In this article, a study on the semi-active systems is being conducted, aiming to compare the main types of such devices, by considering several specific evaluation criteria (such as functional aspects, adaptability, maintenance or cost). At the same time, an improved control strategy is proposed, whose usefulness is verified by simulation in a virtual environment.

\section{Types of semi-active suspensions}

The literature reveals three basic types of suspensions with semi-active control: devices that control the suspension damping (dissipated energy) - these devices allow controlled dissipation of the dissipation capacity so that the response of the mechanically controlled excitation system is minimal; devices that control the rigidity of the system - these devices modify the own frequency of the mechanically controlled system depending on the excitation frequency, to eliminate the possibility that the controlled system resonates; devices that control the inertial mass of the mechanical system - these devices modify the own frequency of the controlled mechanical system according to the excitation frequency and / or response frequency of the system by coupling or decoupling additional inertial masses.

For all types of semi-active systems, control forces are generated by the movement of the mechanically controlled system, similar to passive systems. Damping, rigidity or inertial mass of the controlled suspension are selected in real time, similar to the active control systems - by feedback, based on information retrieved from a sensor system that measures excitation level and / or response level of the controlled system.

The adaptability of semi-active systems is determined by how control is performed. Since systems that control stiffness and inertial mass respectively require sequential control, they are less used in vibration control. In these terms, for the semi-active suspension of automobile wheels and/or axles, the solution with controlled damping device is frequently used. In this respect, the following types of semi-active devices can be systematized: dampers with variable orifices, similar to those used in aircraft landing trains; dampers with controllable fluids (magnetorheological and electrorheological fluids); dampers with controllable friction.

Basically, a damper with variable orifices includes a cylinder containing a cavity whose dimension varies along the axis of the cylinder, axially located between two adjacent cavities larger than the intermediate portion [22]. The plunger head has a surface whose cross-section varies along the axis of the cylinder and is adapted to pass through the variable portion cavity, the plunger head and the cavity defining a variable size orifice between them. The piston rod has a bore that contains a separator driven by the fluid passing through the orifice. Various solutions can be used to modify the size of the holes in accordance with the viscous fluid pressure changes inside the cylinder chambers.

In the case of dampers with controlled friction, the modification of the dissipative characteristic is achieved by altering the normal contact force between the elements of the friction coupler $[6,9,19]$. Such a device is able to dissipate a large amount of energy, the rubbing between the sliding surfaces generating an additional damping that reduce the oscillation/vibration amplitudes. Most frictioncontrolled shock absorbers produce a rectangular, stable hysteretic curve with negligible attenuation. There is a wide variety of such shock absorbers with various materials used for friction surfaces. The dampers with controlled friction have the advantage that can be ordered to operate in a variety of modes, emulating rigidity (similar to a spring) and pseudo-viscous characteristics. On the other hand, these dampers, as well as those with variable orifices, require the use of servomotors (electro- 
hydraulic or electro-mechanical), which involves additional reliability and maintenance problems. In addition, in the case of controlled friction dampers a major problem is mechanical wear of the contact surfaces as well as surface imperfections.

The magnetorheological (MR) and electrorheological (ER) dampers are based on the ability of these fluids to reversibly transform from a fluid-flowing linear fluid into a semi-solid material with controlled flow voltage $[12,15,16,18,20,21,26]$. This transformation occurs when a magnetic or electric field acts on the fluid, resulting in a controlled change in damping forces. The electrical power required to modulate the magnetic or electric field is not very high (meaning low consumption), being able to achieve a wide range of damping forces. The road profile is read by a sensor, which can be a photo or laser camera, the information being processed to determine the required level of damping. Simultaneously, another sensor (usually LVDT - Linear Variable Differential Transformer) determines the current damping rate. On the basis of this information, the control system will generate the command signal for the magnetic/electric field. Finally, the damper will produce a damping level corresponding to the measured road profile. Such a device is semi-active because it can dissipate energy, but does not inject external energy into the system.

\section{Conceptual design of the semi-active suspensions}

For the comparative assessment of the semi-active suspensions, which is useful for selecting the solution for a particular case/vehicle, a conceptual design technique based on multi-criteria analysis was used, by considering the following evaluation criteria: the complexity of the system, in terms of materials, manufacturing and implementation (C1); the flexibility of application to various mechanisms and suspension types (C2); the adaptability to various car running regimes (C3); the easiness of assembly and replacement (C4); the possibility to achieve a wide range of dissipative characteristics (C5); the stability and robustness of the system in response to various external disturbances (C6); the amount of energy required to control and monitor the operation (C7); the changes in system operation due to long-term use, wear, fluid settling (where applicable), and others (C8); the system maintenance (C9); the cost of the system (C10).

The determination of the weight of the evaluation criteria was based on the weighting coefficients. The weight of the criterion is set on a grid with three values (more important - "1", equally important " 0.5 ", less important - "0"). For this, a square table is created, having both lines and columns, the respective $\mathrm{N}$ criteria. In this table, each criterion is compared with each, making the entry on a line and the exit to a column. The points of each criterion are summed up along the line, thus establishing the level of the criterion (place of ranking) relative to the others.

The level value coincides with the place occupied in the criteria ranking. If two criteria get the same number of points, the level will be the semi-sum of the successive places of the two criteria in the criteria ranking. The weighting coefficients $\left(\gamma_{\mathrm{i}}\right)$ can be calculated with different formulas. Frequently used, world-recognized as the most advanced, is the FRISCO formula, according to which:

$$
\gamma_{i}=\frac{p+\Delta p+m+0.5}{-\Delta p^{\prime}+\frac{N}{2}},
$$

where: $\mathrm{p}$ is the sum of the points (per line) obtained by the element taken into account; $\Delta \mathrm{p}$ - the difference between the score of the element taken into account and the score of the element at the last level (if the element taken into account is even the one located on the last level, $\Delta \mathrm{p}$ will be 0 ); $\mathrm{m}$ - the number of criteria exceeded (as score) by the criterion taken into account, i.e. the difference between the level of the last criterion and the level of the criterion taken into account (if two criteria are equal to the last level, then $\mathrm{m}=0.5$ ); $\mathrm{N}$ - the number of criteria; $\Delta \mathrm{p}^{\prime}$ - the difference between the score of the element taken into account and the score of the first element (if the element taken into account is located on the first level, $\Delta \mathrm{p}^{\prime}$ will be 0 ). In these terms, the values shown in Table 1 have been obtained. 
Table 1. The weighting coefficients of the evaluation criteria.

\begin{tabular}{|c|c|c|c|c|c|c|c|c|c|c|c|c|c|}
\hline Criterion & C1 & C2 & C3 & C4 & C5 & C6 & C7 & C8 & C9 & C10 & Points & Level & $\gamma_{\mathrm{i}}$ \\
\hline C1 & 0.5 & 0.5 & 1 & 0 & 0 & 1 & 0 & 0 & 0 & 0 & 3 & 8 & 0.92 \\
\hline C2 & 0.5 & 0.5 & 1 & 1 & 0.5 & 1 & 0 & 0 & 0.5 & 0 & 5 & 5 & 1.78 \\
\hline C3 & 0 & 0 & 0.5 & 0 & 0 & 0.5 & 0 & 0 & 0.5 & 0.5 & 2 & 10 & 0.57 \\
\hline C4 & 1 & 0 & 1 & 0.5 & 1 & 1 & 1 & 0.5 & 0.5 & 0.5 & 7 & 3 & 2.86 \\
\hline C5 & 1 & 0.5 & 1 & 0 & 0.5 & 0.5 & 0 & 0 & 1 & 1 & 5.5 & 4 & 2.19 \\
\hline C6 & 0 & 0 & 0.5 & 0 & 0.5 & 0.5 & 0 & 0 & 0.5 & 0.5 & 2.5 & 9 & 0.73 \\
\hline C7 & 1 & 1 & 1 & 0 & 1 & 1 & 0.5 & 0.5 & 1 & 1 & 8 & 2 & 3.67 \\
\hline C8 & 1 & 1 & 1 & 0.5 & 1 & 1 & 0.5 & 0.5 & 1 & 1 & 8.5 & 1 & 4.7 \\
\hline C9 & 1 & 0.5 & 0.5 & 0.5 & 0 & 0.5 & 0 & 0 & 0.5 & 0.5 & 4 & 7 & 1.18 \\
\hline C10 & 1 & 1 & 0.5 & 0.5 & 0 & 0.5 & 0 & 0 & 0.5 & 0.5 & 4.5 & 6 & 1.45 \\
\hline
\end{tabular}

The note of importance, also called contribution to a criterion, is an integer (maximum 10), which is allocated to each variation according to each criterion, that is, one variant is analyzed in turn from the point of view of each criterion, until all variants. For semi-active suspension system variants and previously defined rating criteria, the notes of importance are shown in Table 2.

Table 2. The notes of importance.

\begin{tabular}{|c|c|c|c|c|c|c|c|c|c|c|}
\hline Criterion & $\mathrm{C} 1$ & $\mathrm{C} 2$ & $\mathrm{C} 3$ & $\mathrm{C} 4$ & $\mathrm{C} 5$ & C6 & $\mathrm{C} 7$ & $\mathrm{C} 8$ & C9 & $\mathrm{C} 10$ \\
\hline Damper with variable orifices (VO) & 8 & 9 & 6 & 8 & 10 & 8 & 7 & 10 & 10 & 10 \\
\hline Magnetorheological damper (MR) & 7 & 10 & 8 & 9 & 8 & 10 & 8 & 9 & 9 & 10 \\
\hline Electrorheological damper (ER) & 8 & 8 & 4 & 7 & 8 & 6 & 7 & 8 & 8 & 9 \\
\hline Damper with controlled friction $(\mathrm{CF})$ & 10 & 8 & 10 & 10 & 5 & 8 & 8 & 6 & 10 & 10 \\
\hline
\end{tabular}

The calculation of the products between the N-notes and the weighting coefficients is done in a table called the matrix of consequences, finally calculating the scores (amounts) of these products. For the comparative study of semi-active suspensions, the values obtained on the basis of the presented algorithm are shown in Table 3. It is noted that the highest score (177.31) places the semi-active suspension with magnetorheological damper first.

Table 3. The matrix of consequences.

\begin{tabular}{|c|c|c|c|c|c|c|c|c|c|}
\hline \multirow{2}{*}{ Criterion } & \multirow{2}{*}{$\gamma_{\mathrm{i}}$} & \multicolumn{2}{|c|}{ VO } & \multicolumn{2}{|c|}{ MR } & \multicolumn{2}{|c|}{ ER } & \multicolumn{2}{|c|}{$\mathrm{CF}$} \\
\hline & & $\mathrm{N}$ & $\mathrm{N} \cdot \gamma_{\mathrm{i}}$ & $\mathrm{N}$ & $\mathrm{N} \cdot \gamma_{\mathrm{i}}$ & $\mathrm{N}$ & $\mathrm{N} \cdot \gamma_{\mathrm{i}}$ & $\mathrm{N}$ & $\mathrm{N} \cdot \gamma_{\mathrm{i}}$ \\
\hline $\mathrm{C} 1$ & 0.92 & 8 & 7.33 & 7 & 6.44 & 8 & 7.33 & 10 & 9.17 \\
\hline $\mathrm{C} 2$ & 1.78 & 9 & 16 & 10 & 18 & 8 & 14.2 & 8 & 14.2 \\
\hline $\mathrm{C} 3$ & 0.57 & 6 & 3.43 & 8 & 4.6 & 4 & 2.29 & 10 & 5.71 \\
\hline $\mathrm{C} 4$ & 2.86 & 8 & 22.9 & 9 & 26 & 7 & 20 & 10 & 28.6 \\
\hline $\mathrm{C} 5$ & 2.19 & 10 & 21.9 & 8 & 18 & 8 & 17.5 & 5 & 10.9 \\
\hline C6 & 0.73 & 8 & 5.85 & 10 & 7.3 & 6 & 4.38 & 8 & 5.85 \\
\hline $\mathrm{C} 7$ & 3.67 & 7 & 25.7 & 8 & 29 & 7 & 25.7 & 8 & 29.3 \\
\hline $\mathrm{C} 8$ & 4.7 & 10 & 47 & 9 & 42 & 8 & 37.6 & 6 & 28.2 \\
\hline C9 & 1.18 & 10 & 11.8 & 9 & 11 & 8 & 9.45 & 10 & 11.8 \\
\hline $\mathrm{C} 10$ & 1.45 & 10 & 14.5 & 10 & 15 & 9 & 13.1 & 10 & 14.5 \\
\hline \multicolumn{2}{|c|}{ Total } & \multicolumn{2}{|c|}{176.41} & \multicolumn{2}{|c|}{177.31} & \multicolumn{2}{|c|}{151.49} & \multicolumn{2}{|c|}{158.31} \\
\hline
\end{tabular}


Thoth Publishing House

\section{Semi-active suspension control methods}

Regarding the control of the semi-active suspension systems, several strategies are frequently used in literature, such as sky-hook and ground-hook $[8,10,25]$. The sky-hook control technique aims to minimize the vertical oscillations of the sprung mass (i.e. the car body), by connecting it with a virtual damper to the sky, as shown in Figure 2. Obviously, in practice, such a connection is not possible. The sky-hook control strategy improves the comfort performance, but may result in inappropriate maneuverability of the car. The damping force for the sky-hook control can be modeled by the following equations:

$$
F_{s k y}=C_{s k y} \cdot \mathbb{Z}-\text { if } \&(Z \mathbb{Z}-\mathbb{Z}) \geq 0 \text {, }
$$

and respectively

$$
F_{\text {sky }}=C_{\text {min }} \cdot(\&-\&) \text { - if } \&(\&-\mathbb{Z})<0 .
$$

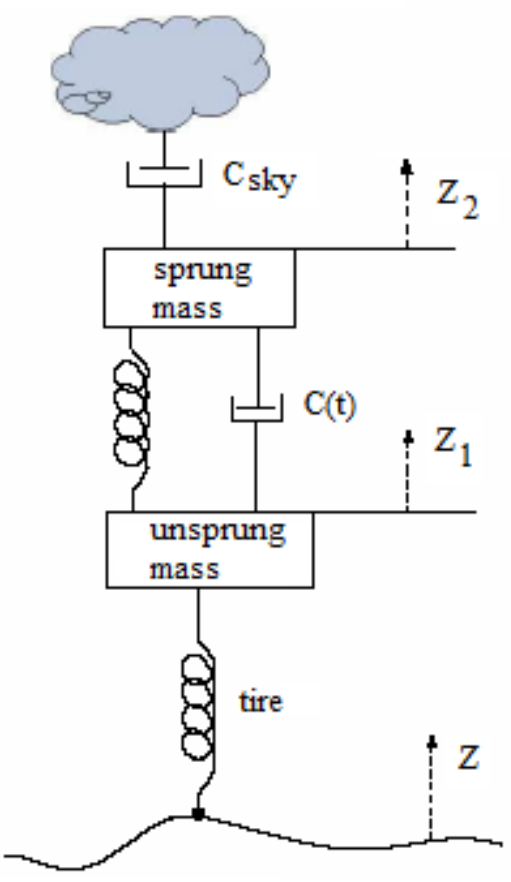

Figure 2. Sky-hook control.

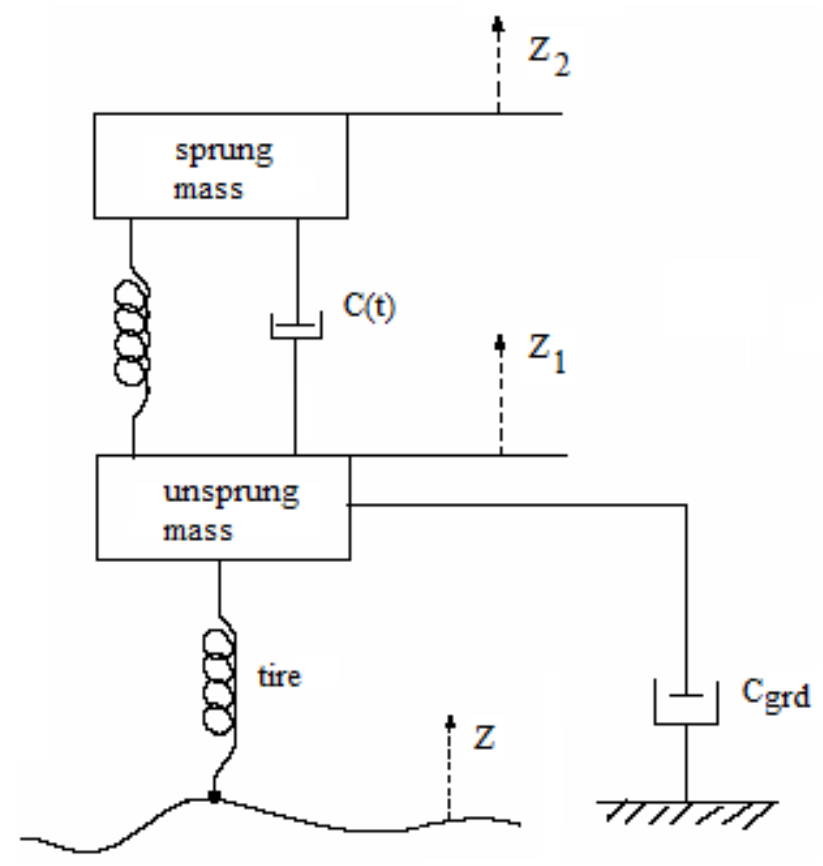

Figure 3. Ground-hook control.

The ground-hook control technique intends to improve the ride performance of the car by reducing the movement on the unsprung mass (wheel or axle), which is connected by a virtual damper to ground (Figure 3). In this case, the damping force can be modeled by the following equations:

$$
F_{g r d}=C_{g r d} \cdot \mathbb{Z}-\text { if }\left(-\mathbb{Z} \cdot\left(\mathbb{Z}_{2}-\mathbb{Z}_{1}^{\mathbb{E}}\right)\right) \geq 0 \text {, }
$$

and respectively

$$
F_{\text {grd }}=C_{\text {min }} \cdot\left(\mathbb{Z}_{2}-\mathbb{Z}_{1}\right) \text { - if }\left(-\mathbb{Z}_{1} \cdot\left(\mathbb{Z}_{2}-\mathbb{Z}_{1}\right)<0\right)
$$


Thoth Publishing House

The combination between the sky-hook and ground-hook techniques defines the so called hybrid control strategy, the corresponding damping force being expressed by the following equation:

$$
F_{\text {hyb }}=C(t) \cdot(\& 2-\&)=\beta \cdot F_{\text {sky }}+(1-\beta) \cdot F_{\text {grd }},
$$

where $C_{\text {sky }}, C_{\text {grd }}>C_{\text {min }}>0, \beta \in[0,1]$, the value of the coefficient $\beta$ determining the individual contribution of the sky-hook and ground-hook techniques into the hybrid strategy.

The sky-hook technique can be also used for controlling the roll or pitch oscillations of the car body, by replacing the translational damper with a rotary virtual damper between car body and sky. For example, Figure 4 shows the sky-hook model for controlling the roll movement, by considering a transversal half-car model, which can be used for both front wheels and rear axle suspensions (see Figure 5).

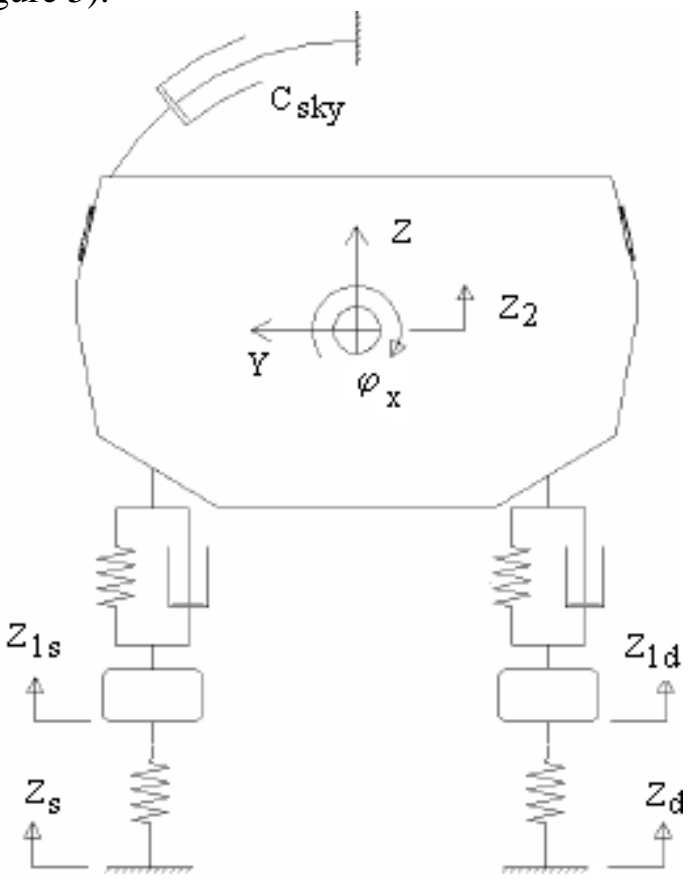

Figure 4. Shy-hook model for the roll movement.

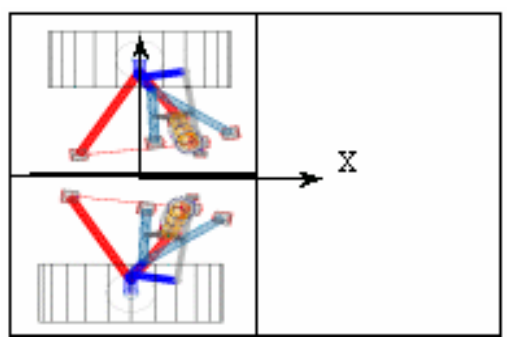

a.

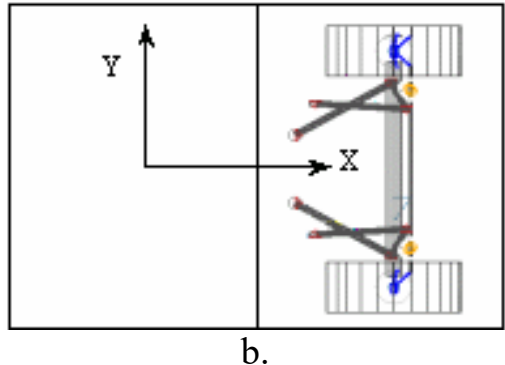

Figure 5. Transversal half-car models for the front wheels (a) and the rear axle (b).

Considering the limitations of the existing techniques, the paper proposes an improved control strategy, by which the road bumps are treated as disturbances to be eliminated by the control system. In this regard, a quarter-car model that corresponds to the wheel suspension system was considered (Figure 6$)$. The model contains two masses $\left(\mathrm{m}_{1}\right.$ unsprung mass, $\mathrm{m}_{2}$ - sprung mass), which are connected by a spring $\left(\mathrm{k}_{2}\right)$ $\&$ damper $\left(c_{2}\right)$ group, the control signal (u) being used to modify the damping coefficient $\left(c_{2}=c_{2(0)} \pm c_{2}(u)\right.$, where $c_{2(0)}$ corresponds to the passive suspension), and consequently the damping force. In addition, $\mathrm{k}_{1}$ and $\mathrm{c}_{1}$ are used to model the tire.

The adaptive suspension is a MIMO (Multi-Input - Multi-Output) system, as follows: inputs - the road disturbance (z) and the command signal (u); outputs - the vertical displacements of the wheel $\left(\mathrm{z}_{1}\right)$ and car body $\left(\mathrm{z}_{2}\right)$. For the control system design, the performance index is related to the relative displacement $z_{2}-z_{1}$, which is the effect of the two input signals, by the principle of overlapping effects.

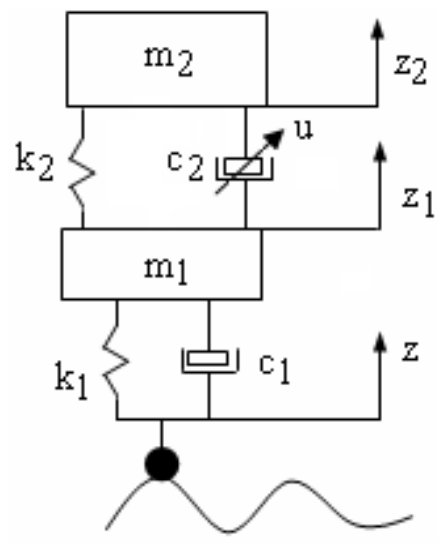

Figure 6. The quarter-car suspension model. 
The two transfer functions $G_{z}$ and $G_{u}$ are determined by using the dynamic equations of motion, based on the Laplace transform, the detailed mathematic algorithm following to be featured in a future paper. With the aim to simplify the controller synthesis, the control scheme was successively transformed by using the diagram algebra. Thus, the transfer function of the controlled system is $\mathrm{G}_{\mathrm{U}}$, while the perturbation is filtered by the new function $\mathrm{G}_{\mathrm{F}}(\mathrm{s})=\mathrm{G}_{\mathrm{Z}} / \mathrm{G}_{\mathrm{U}}$, as shown in Figure 7.

The proposed adaptive suspension system was modeled - implemented by using a virtual prototyping software platform, which contains MBS (Multi-Body Systems) and DFC (Design for Control) software solutions, namely ADAMS - for modeling the mechanical device, and EASY5 - for modeling the control system. The MBS and DFC models have been integrated in mechatronic concept, the communication being assured through the input \& output plants, which are managed by the plug-in ADAMS/Controls.

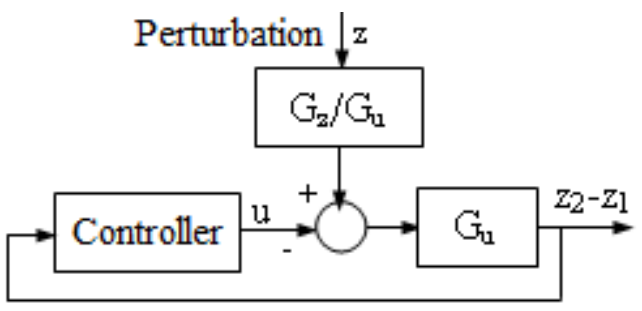

Figure 7. The proposed control strategy.

The application for this paper is performed for a half-car model, which corresponds to a rear beam axle suspension system (Figure 8). In relative motion to car body, the rear axle is guided by a guiding mechanism with four links, which are connected to the adjacent bodies by bushings (compliant joints). The spring \& damper groups are concentrically disposed towards the axle ends. The damping forces of the shock absorbers are adjusted in accordance with the proposed control strategy.

The virtual model was analyzed in the passing over bumps regime (Figure 9), by simulating the dynamic test on virtual stand. The front axle suspension was replaced by a spherical joint between car body and ground, placed on the longitudinal axis of the car at the front wheels level [24]. The road profile on the two rear wheels was materialized by the motion laws imposed to the linear actuators on which the wheels are anchored. It was considered that the left wheel passes a bump with the amplitude of $80 \mathrm{~mm}$, while the right wheel runs on the smooth surface, the right actuator remaining fixed, as shown in Figure 10. The motion law for the left actuator was modeled by using a time (t) conditional function [29], with the following expression:

$$
I F(t-0.1: 0,0,80 \cdot \sin (\pi \cdot t / 0.05))+I F(t-0.15: 0,0,-80 \cdot \sin (\pi \cdot t / 0.05)) .
$$

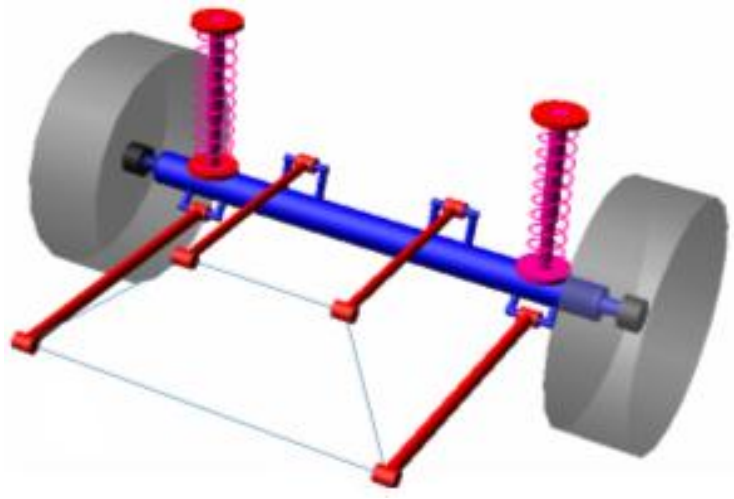

Figure 8. The MBS model of the rear axle suspension system.

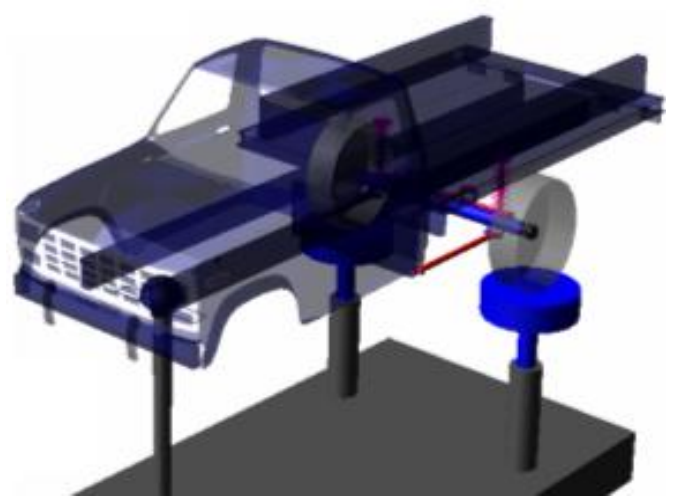

Figure 9. The virtual stand for simulating the passing over bumps.

For each of the two shock absorbers from the semi-active suspension system, the control block diagram developed in EASY5 is the one shown in Figure 11, where ADAMS Mechanism is the interface block that addresses the MBS mechanical device model (conceived in ADAMS). The control system is based on PID (Proportional-Integral-Derivative) controller, which corrects the difference between the imposed and measured values by computing and applying a compensatory measure that 
Thoth Publishing House

adapts the system properly $[7,11]$. In EASY5, this type of controller is defined by the following three parameters (which are independent design variables in the optimal design of the controller): proportional control gain (P), integration control gain (I), and derivative action time constant (D) [28]. The optimization of the controller was performed by using the scripting capabilities integrated in EASY5 - MAT (Matrix Algebra Tool), the control system model being managed as an EMX function, according to the optimization procedure presented in $[3,5]$.

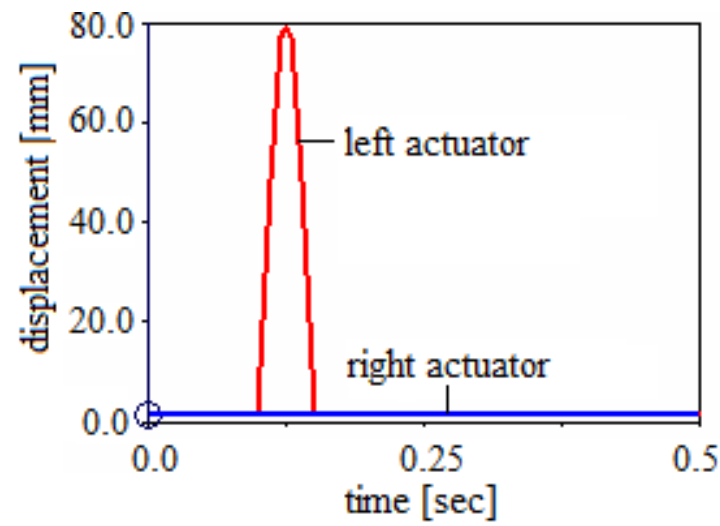

Figure 10. The vertical displacement of the left and right actuators.

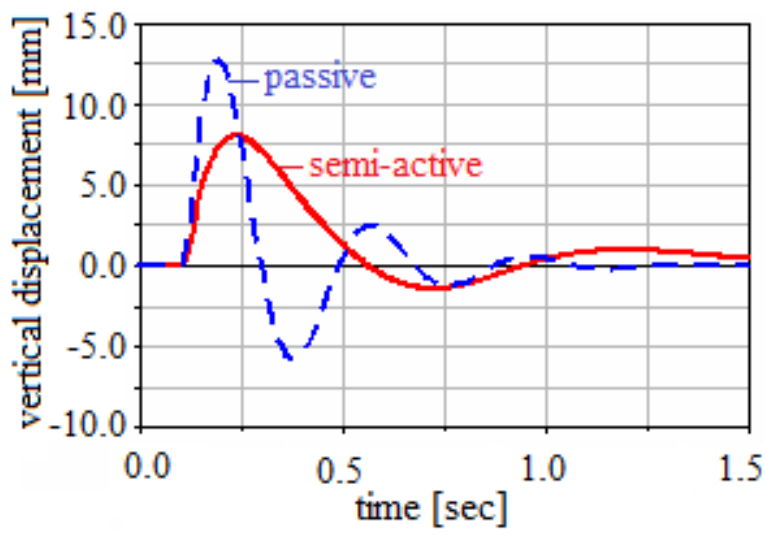

a.

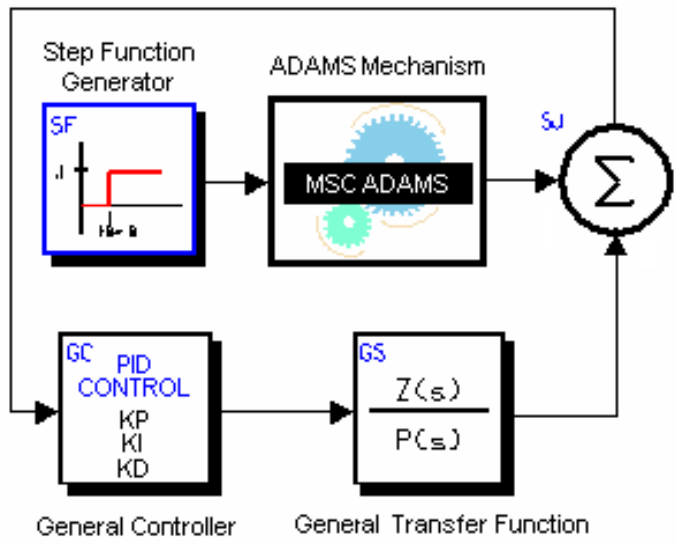

Figure 11. The control system model.

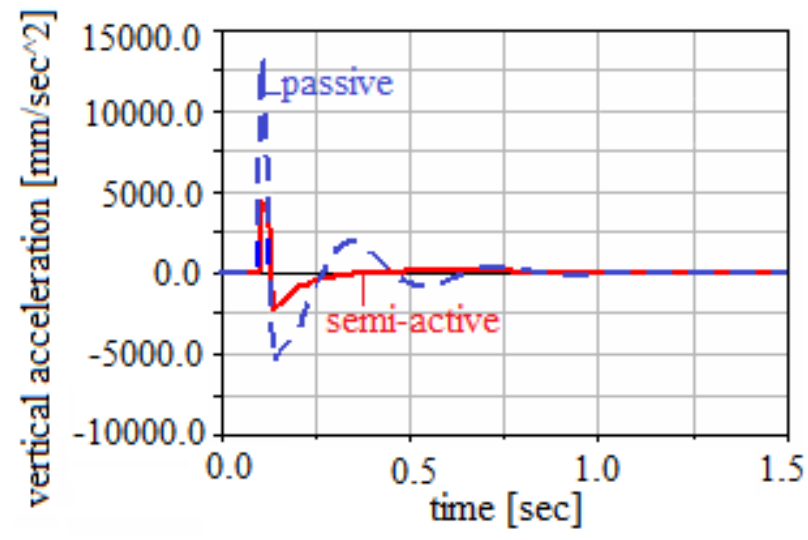

b.

Figure 12. The vertical displacement (a) and acceleration (b) of the car body.

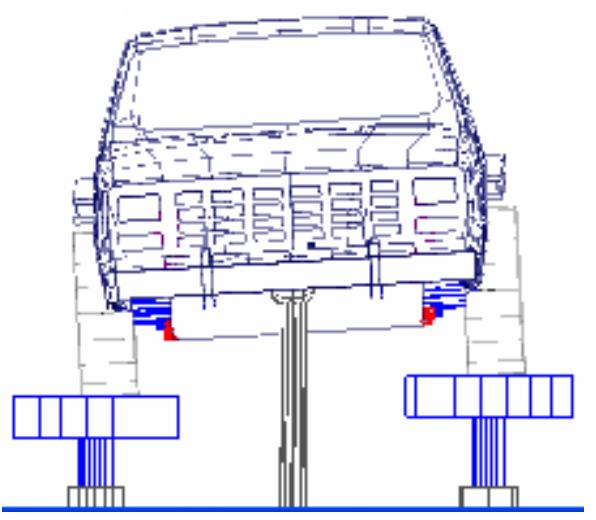

a.

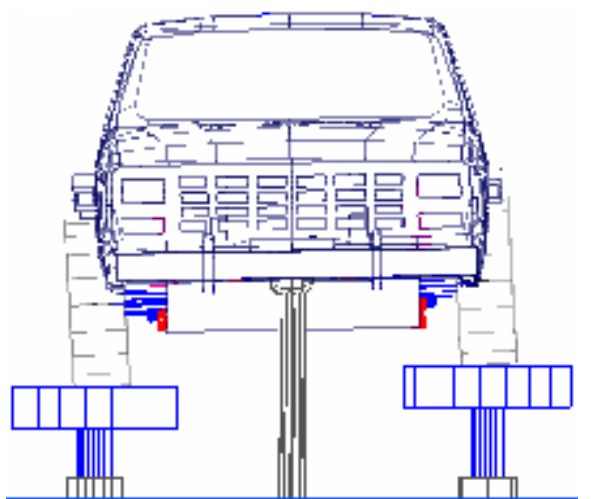

b.

Figure 13. Graphical simulation frames for passive (a) and semi-active (b) suspension. Results and conclusions 
Among the results of the dynamic analysis carried out by the co-simulation of the MBS (ADAMS) and DFC (EASY5) models, Figure 12 shows the time-history variations of the vertical displacement and acceleration of the car body, which are some of the most important factors for the vehicle comfort performance. The results correspond to both the classic variant of passive suspension system, as well as the semi-active suspension system adjusted by the previously presented control strategy. The amplitude of the vertical oscillations and their damping time are lower in the case of the semi-active suspension, thus proving the improved behavior provided by the proposed solution. The improvement of the dynamic behavior of the vehicle, mainly in terms of roll oscillations, can be also observed in the graphical simulation frames shown in Figure 13.

The use of virtual prototyping platforms, such as that used in this work, brings important benefits in the design process of the mechanical and mechatronic systems, which focuses on reducing the costs and time of the design \& development process, while increasing the quality (working performances) of the product, in this case the semi-active suspension system of the motor vehicles. Although it does not ensure the performance of an active suspension (which on the other hand is even more complex and expensive), the semi-active suspension system allows improving the dynamic behavior of the vehicle compared to the classic passive suspension without requiring external force generating elements, but by the appropriate modification of the damping characteristic of the shock absorber according to the road profile (irregularities). Considering the road irregularities as disturbances whose effect must be minimized by the control system allows a better efficiency of the suspension system. A more detailed study (including a larger palette of dynamic analysis/simulation results) will be presented in a forthcoming paper, in which the robustness and stability of the proposed control system will be also addressed.

\section{References}

[1] Alexandru C, Alexandru P 2010 The virtual prototype of a mechatronic suspension system with active force control WSEAS Transactions on Systems 9(9) pp 927-936

[2] Alexandru C, Alexandru P 2010 Dynamic modeling and simulation in virtual environment of an active suspension system Proceedings of the 14th WSEAS International Conference on Systems 1 pp 81-86

[3] Alexandru C, Alexandru P 2011 Control strategy for an active suspension system World Academy of Science, Engineering and Technology 79 pp 126-131

[4] Alexandru C, Alexandru P 2011 A comparative analysis between the vehicles' passive and active suspensions International Journal of Mechanics 5(4) pp 371-378

[5] Alexandru C 2012 The optimal design of the mechanical systems using parametric technique \& MBS (Multi-Body Systems) software Advanced Materials Research 463-464 pp 1129-1132

[6] Carlson D 2001 Magnetically controled friction damper Patent nr. WO 01/73313 A2

[7] Dorf RC, Bishop RH 2016 Modern Control Systems (Pearson)

[8] Duysinx P, Bruls O, Collard JF, Fisette P, Lauwerys C, Swevers J 2005 Optimization of mechatronic systems: application to a modern car equipped with a semi-active suspension Proceedings of the 6th World Congresses of Structural and Multidisciplinary Optimization pp 1-10

[9] Guglielmino E, Edge K 2004 A controlled friction damper for vehicle applications Control Engineering Practice 12 pp 431-443

[10] Guglielmino E, Sireteanu T, Stammers C, Ghita G, Giuclea M 2008 Semi-Active Suspension Control. Improved Vehicle Ride and Road Friendliness (London: Springer-Verlag)

[11] Haque HH, Hassan HM, Hossain SM 2014 Comparison of control system using PLC \& PID Proceedings of the ASEE 2014 Conference pp 99(1-6)

[12] Holzmann K, Kemmetmuller W, Kugi A, Stork M 2006 Design, mathematical modeling and control of an asymmetrical electrorheological damper Proceedings of the 4th IFAC Symposium on Mechatronic Systems pp 372-377

[13] Hyniova K, Stribrsky A, Honcu J, Kruczek A 2009 Active suspension system with linear 
Thoth Publishing House

electric motor WSEAS Transactions on Systems 8(2) pp 278-287

[14] Hyvärinen J P 2004 The Improvement of Full Vehicle Semi-Active Suspension Through Kinematical Model (University of Oulu)

[15] Kemmetmüller W, Kugi A 2004 Modeling and control of an electrorheological actuator Proceedings of the 3-rd IFAC Symposium on Mechatronic Systems pp 271-276

[16] Kugi A, Holzmann K, Kemmetmüller W 2005 Active and semi-active control of electrorheological fluid devices Proceedings of the IUTAM Symposium on Vibration Control of Nonlinear Mechanisms and Structures pp 203-212

[17] Levesley M C, Ramli R, Stembridge N, Crolla D A 2007 Multibody cosimulation of semiactive suspension systems Proceedings of the Institution of Mechanical Engineers, Part K: Journal of Multi-Body Dynamics 221(1) pp 99-115

[18] Li T, Zhang F, Ito M 2002 Semi-active suspension system with electro-rheological damper Proceedings of the 6th WSEAS International Conference on Circuits, Systems, Communications and Computers pp 6971-6974

[19] Lopez I, Nijmeijer H 2009 Prediction and validation of the energy dissipation of a friction damper Journal of Sound and Vibration 328 pp 396-410

[20] Mukhlis R Z, Mohamed N A, Nor M J 2006 The design and simulation of flow mode electrorheological damper Jurnal Teknologi 44(A) pp 115-125

[21] Poynor J 2003 Innovative Designs for Magneto-Rheological Dampers (Virginia Polytechnic Institute and State University)

[22] Ralph H 1994 Variable oriffice oil/gas damper US Patent 5.325.943

[23] Sharp R, Hassan S 1986 An evaluation of passive automotive suspension systems with variable stiffness and damping parameters Vehicle Systems Dynamics 15 pp 335-350.

[24] Țoțu V, Alexandru C 2014 Dynamic simulation of a motor vehicle in virtual prototyping environment Applied Mechanics and Materials 555 pp 369-374

[25] Zhang H, Winner H, Li W 2009 Comparison between skyhook and minimax control strategies for semi-active suspension system World Academy of Science, Engineering and Technology 55 pp 624-627

[26] Zhu X, Jing X, Cheng L 2012 Magnetorheological fluid dampers: A review on structure design and analysis Journal of Intelligent Material Systems and Structures 23(8) $839-873$

[27] Wang J, Wilson D, Xu W, Crolla D 2005 Active suspension control to improve vehicle ride and steady-state handling Proceedings of the 44th IEEE Conference on Decision and Control pp 1982-1987

[28] *** 2005 EASY5 Guide Help (MSC Software Press)

[29] *** 2005 Getting Started using ADAMS (MSC Software Press) 